Research Article

\title{
Existence and Multiplicity of Solutions for Some Fractional Boundary Value Problem via Critical Point Theory
}

\author{
Jing Chen ${ }^{1,2}$ and X. H. Tang ${ }^{1}$ \\ ${ }^{1}$ School of Mathematical Sciences and Computing Technology, Central South University, Changsha, \\ Hunan 410083, China \\ ${ }^{2}$ School of Mathematics and Computing Sciences, Hunan University of Science and Technology, Xiangtan, \\ Hunan 411201, China
}

Correspondence should be addressed to X. H. Tang, tangxh@mail.csu.edu.cn

Received 18 October 2011; Accepted 27 November 2011

Academic Editor: Kanishka Perera

Copyright (C) 2012 J. Chen and X. H. Tang. This is an open access article distributed under the Creative Commons Attribution License, which permits unrestricted use, distribution, and reproduction in any medium, provided the original work is properly cited.

We study the existence and multiplicity of solutions for the following fractional boundary value problem: $(d / d t)\left((1 / 2)_{0} D_{t}^{-\beta}\left(u^{\prime}(t)\right)+(1 / 2)_{t} D_{T}^{-\beta}\left(u^{\prime}(t)\right)\right)+\nabla F(t, u(t))=0$, a.e. $t \in[0, T], u(0)=u(T)=$ 0 , where $F(t, \cdot)$ are superquadratic, asymptotically quadratic, and subquadratic, respectively. Several examples are presented to illustrate our results.

\section{Introduction and Main Results}

Consider the fractional boundary value problem (BVP for short) of the following form:

$$
\begin{gathered}
\frac{d}{d t}\left(\frac{1}{2}{ }_{0} D_{t}^{-\beta}\left(u^{\prime}(t)\right)+\frac{1}{2}{ }_{t} D_{T}^{-\beta}\left(u^{\prime}(t)\right)\right)+\nabla F(t, u(t))=0, \quad \text { a.e. } t \in[0, T], \\
u(0)=u(T)=0,
\end{gathered}
$$

where ${ }_{0} D_{t}^{-\beta}$ and ${ }_{t} D_{T}^{-\beta}$ are the left and right Riemann-Liouville fractional integrals of order $0 \leq \beta<1$, respectively, $F:[0, T] \times \mathbb{R}^{N} \rightarrow \mathbb{R}$ satisfies the following assumptions.

(A) $F(t, x)$ is measurable in $t$ for every $x \in \mathbb{R}^{N}$ and continuously differentiable in $x$ for a.e. $t \in[0, T]$, and there exist $a \in C\left(\mathbb{R}^{+}, \mathbb{R}^{+}\right), b \in L^{1}\left(0, T ; \mathbb{R}^{+}\right)$, such that

$$
|F(t, x)| \leq a(|x|) b(t), \quad|\nabla F(t, x)| \leq a(|x|) b(t),
$$


for all $x \in \mathbb{R}^{N}$ and a.e. $t \in[0, T]$. In particular, if $\beta=0$, BVP (1.1) reduces to the standard second-order boundary value problem of the following form:

$$
\begin{gathered}
u^{\prime \prime}(t)+\nabla F(t, u(t))=0, \quad \text { a.e. } t \in[0, T] \\
u(0)=u(T)=0 .
\end{gathered}
$$

Differential equations with fractional order are generalization of ordinary differential equations to noninteger order. This generalization is not mere mathematical curiosities but rather has interesting applications in many areas of science and engineering such as in viscoelasticity, electrical circuits, and neuron modeling. The need for fractional order differential equations stems in part from the fact that many phenomena cannot be modeled by differential equations with integer derivatives. Such differential equations got the attention of many researchers and considerable work has been done in this regard, see the monographs of Kilbas et al. [1], Miller and Ross [2], Podlubny [3], Samko et al. [4], and the papers [5-20] and the references therein.

Recently, there are many papers dealing with the existence of solutions (or positive solutions) of nonlinear initial (or singular and nonsingular boundary) value problems of fractional differential equation by the use of techniques of nonlinear analysis (fixed-point theorems [12-14], Leray-Schauder theory [15, 16], lower and upper solution method, monotone iterative method [17-19], Adomian decomposition method [20], etc.), see [12-20] and the references therein.

Variational methods are very powerful techniques in nonlinear analysis and are extensively used in many disciplines of pure and applied mathematics including ordinary and partial differential equations, mathematical physics, gauge theory, and geometrical analysis. The existence and multiplicity of solutions for Hamilton systems, Schrödinger equations, and Dirac equations have been studied extensively via critical point theory, see [21-34].

In [32], Jiao and Zhou obtained the existence of solutions for BVP (1.1) by Mountain Pass theorem under the Ambrosetti-Rabinowitz condition (denoted by A.R. condition). Under the usual A.R. condition, it is easy to show that the energy functional associated with the system has the Mountain Pass geometry and satisfies the (PS) condition. However, the A.R. condition is so strong that many potential functions cannot satisfy it, then the problem becomes more delicate and complicated.

In this paper, in order to establish the existence and multiplicity of solutions for BVP (1.1) under distinct hypotheses on potential function by critical point theory, we introduce some functional space $E^{\alpha}$, where $\alpha \in(1 / 2,1]$, and divide the problem into the following three cases.

\subsection{The Superquadratic Case}

For the superquadratic case, we make the following assumptions.

(A1) $\lim _{|x| \rightarrow 0} F(t, x) /|x|^{2}=0, \liminf _{|x| \rightarrow \infty} F(t, x) /|x|^{2} \geq L>\pi^{2} /|\cos (\pi \alpha)| \Gamma^{2}(2-\alpha) T^{2 \alpha}(3-$ $2 \alpha$ ) uniformly for some $L>0$ and a.e. $t \in[0, T]$.

(A2) $\lim \sup _{|x| \rightarrow+\infty} F(t, x) /|x|^{r} \leq M<+\infty$ uniformly for some $M>0$ and a.e. $t \in[0, T]$. 
(A3) $\liminf _{|x| \rightarrow+\infty}((\nabla F(t, x), x)-2 F(t, x)) /|x|^{\mu} \geq Q>0$ uniformly for some $Q>0$ and a.e. $t \in[0, T]$,

where $r>2$ and $\mu>r-2$. We state our first existence result as follows.

Theorem 1.1. Assume that (A1)-(A3) hold and that $F(t, x)$ satisfies the condition (A). Then BVP (1.1) has at least one solution on $E^{\alpha}$.

\subsection{The Asymptotically Quadratic Case}

For the asymptotically quadratic case, we assume the following.

$\left(\mathrm{A}^{\prime}\right) \lim \sup _{|x| \rightarrow+\infty} F(t, x) /|x|^{2} \leq M<+\infty$ uniformly for some $M>0$ and a.e. $t \in[0, T]$.

(A4) There exists $\tau(t) \in L^{1}\left(0, T ; \mathbb{R}^{+}\right)$such that $(\nabla F(t, x), x)-2 F(t, x) \geq \tau(t)$ for all $x \in \mathbb{R}^{N}$ and a.e. $t \in[0, T]$.

(A5) $\lim _{|x| \rightarrow+\infty}[(\nabla F(t, x), x)-2 F(t, x)]=+\infty$ for a.e. $t \in[0, T]$.

Our second and third main results read as follows.

Theorem 1.2. Assume that $F(t, x)$ satisfies $(A),(A 1),\left(A 2^{\prime}\right),(A 4)$, and (A5). Then BVP (1.1) has at least one solution on $E^{\alpha}$.

Theorem 1.3. Assume that $F(t, x)$ satisfies $(A),(A 1),\left(A 2^{\prime}\right)$, and the following conditions:

(A4') there exists $\tau(t) \in L^{1}\left(0, T ; \mathbb{R}^{+}\right)$such that $(\nabla F(t, x), x)-2 F(t, x) \leq \tau(t)$ for all $x \in \mathbb{R}^{N}$ and a.e. $t \in[0, T]$;

$\left(\mathrm{A} 5^{\prime}\right) \lim _{|x| \rightarrow+\infty}[(\nabla F(t, x), x)-2 F(t, x)]=-\infty$ for a.e. $t \in[0, T]$.

Then BVP (1.1) has at least one solution on $E^{\alpha}$.

\subsection{The Subquadratic Case}

For the subquadratic case, we give the following multiplicity result.

Theorem 1.4. Assume that $F(t, x)$ satisfies the following assumption:

(A6) $F(t, x):=a(t)|x|^{\gamma}$, where $a(t) \in L^{\infty}\left(0, T ; \mathbb{R}^{+}\right)$and $1<\gamma<2$ is a constant.

Then BVP (1.1) has infinitely many solutions on $E^{\alpha}$.

\section{Preliminaries}

In this section, we recall some background materials in fractional differential equation and critical point theory. The properties of space $E^{\alpha}$ are also listed for the convenience of readers. 
Definition 2.1 (see [1]). Let $f(t)$ be a function defined on $[a, b]$ and $q>0$. The left and right Riemann-Liouville fractional integrals of order $q$ for function $f(t)$ denoted by ${ }_{a} D_{t}^{-q} f(t)$ and ${ }_{t} D_{b}^{-q} f(t)$, respectively, are defined by

$$
\begin{aligned}
& { }_{a} D_{t}^{-q} f(t)=\frac{1}{\Gamma(q)} \int_{a}^{t}(t-s)^{q-1} f(s) d s, \\
& { }_{t} D_{b}^{-q} f(t)=\frac{1}{\Gamma(q)} \int_{t}^{b}(t-s)^{q-1} f(s) d s,
\end{aligned}
$$

provided the right-hand sides are pointwise defined on $[a, b]$, where $\Gamma$ is the gamma function.

Definition 2.2 (see [1]). Let $f(t)$ be a function defined on $[a, b]$ and $q>0$. The left and right Riemann-Liouville fractional derivatives of order $q$ for function $f(t)$ denoted by ${ }_{a} D_{t}^{q} f(t)$ and ${ }_{t} D_{b}^{q} f(t)$, respectively, are defined by

$$
\begin{gathered}
{ }_{a} D_{t}^{q} f(t)=\frac{d^{n}}{d t^{n}}{ }_{a} D_{t}^{q-n} f(t)=\frac{1}{\Gamma(n-q)} \frac{d^{n}}{d t^{n}}\left(\int_{a}^{t}(t-s)^{n-q-1} f(s) d s\right), \\
{ }_{t} D_{b}^{q} f(t)=(-1)^{n} \frac{d^{n}}{d t^{n}}{ }_{t} D_{b}^{q-n} f(t)=\frac{1}{\Gamma(n-q)}(-1)^{n} \frac{d^{n}}{d t^{n}}\left(\int_{t}^{b}(s-t)^{n-q-1} f(s) d s\right),
\end{gathered}
$$

where $t \in[a, b], n-1 \leq q<n$ and $n \in \mathbb{N}$.

The left and right Caputo fractional derivatives are defined via the above RiemannLiouville fractional derivatives. In particular, they are defined for the function belonging to the space of absolutely continuous functions, which we denote by $A C\left([a, b], \mathbb{R}^{N}\right)$. $A C^{k}\left([a, b], \mathbb{R}^{N}\right)(k=1, \ldots)$ is the space of functions $f$ such that $f \in C^{k-1}\left([a, b], \mathbb{R}^{N}\right)$ and $f^{(k-1)} \in A C\left([a, b], \mathbb{R}^{N}\right)$. In particular, $A C\left([a, b], \mathbb{R}^{N}\right)=A C^{1}\left([a, b], \mathbb{R}^{N}\right)$.

Definition 2.3 (see [1]). Let $q \geq 0$ and $n \in \mathbb{N}$. If $q \in[n-1, n)$ and $f(t) \in A C^{n}\left([a, b], \mathbb{R}^{N}\right)$, then the left and right Caputo fractional derivative of order $q$ for function $f(t)$ denoted by ${ }_{a}^{c} D_{t}^{q} f(t)$ and ${ }_{t}^{c} D_{b}^{q} f(t)$, respectively, exist almost everywhere on $[a, b] .{ }_{a}^{c} D_{t}^{q} f(t)$ and ${ }_{t}^{c} D_{b}^{q} f(t)$ are represented by

$$
\begin{gathered}
{ }_{a}^{c} D_{t}^{q} f(t)={ }_{a} D_{t}^{q-n} f^{(n)}(t)=\frac{1}{\Gamma(n-q)}\left(\int_{a}^{t}(t-s)^{n-q-1} f^{(n)}(s) d s\right), \\
{ }_{t}^{c} D_{b}^{q} f(t)=(-1)^{n} D_{b}^{q-n} f^{(n)}(t)=\frac{(-1)^{n}}{\Gamma(n-q)}\left(\int_{t}^{b}(s-t)^{n-q-1} f^{(n)}(s) d s\right),
\end{gathered}
$$

respectively, where $t \in[a, b]$. 
Property 2.4 (see [1]). The left and right Riemann-Liouville fractional integral operators have the property of a semigroup, that is,

$$
{ }_{a} D_{t}^{-q_{1}}\left({ }_{a} D_{t}^{-q_{2}} f(t)\right)={ }_{a} D_{t}^{-q_{1}-q_{2}} f(t), \quad{ }_{t} D_{b}^{-q_{1}}\left({ }_{t} D_{b}^{-q_{2}} f(t)\right)={ }_{t} D_{b}^{-q_{1}-q_{2}} f(t), \quad \forall q_{1}, q_{2}>0 .
$$

Definition 2.5 (see [32]). Define $0<\alpha \leq 1$ and $1<p<\infty$. The fractional derivative space $E_{0}^{\alpha, p}$ is defined by the closure of $C_{0}^{\infty}\left([0, T], \mathbb{R}^{N}\right)$ with respect to the norm

$$
\|u\|_{\alpha, p}=\left(\int_{0}^{T}|u(t)|^{p} d t+\int_{0}^{T}\left|{ }_{0}^{c} D_{t}^{\alpha} u(t)\right|^{p} d t\right)^{1 / p}, \quad \forall u \in E_{0}^{\alpha, p}
$$

where $C_{0}^{\infty}\left([0, T], \mathbb{R}^{N}\right)$ denotes the set of all functions $u \in C^{\infty}\left([0, T], \mathbb{R}^{N}\right)$ with $u(0)=u(T)=0$. It is obvious that the fractional derivative space $E_{0}^{\alpha, p}$ is the space of functions $u \in L^{p}\left(0, T ; \mathbb{R}^{N}\right)$ having an $\alpha$-order Caputo fractional derivative ${ }_{0}^{c} D_{t}^{\alpha} u \in L^{p}\left(0, T ; \mathbb{R}^{N}\right)$ and $u(0)=u(T)=0$.

Proposition 2.6 (see [32]). Let $0<\alpha \leq 1$ and $1<p<\infty$. The fractional derivative space $E_{0}^{\alpha, p}$ is a reflexive and separable space.

Proposition 2.7 (see [32]). Let $0<\alpha \leq 1$ and $1<p<\infty$. For all $u \in E_{0}^{\alpha, p}$, one has

$$
\|u\|_{L^{p}} \leq \frac{T^{\alpha}}{\Gamma(\alpha+1)}\left\|_{0}^{c} D_{t}^{\alpha} u\right\|_{L^{p}}
$$

Moreover, if $\alpha>1 / p$ and $1 / p+1 / q=1$, then

$$
\|u\|_{\infty} \leq \frac{T^{\alpha-1 / p}}{\Gamma(\alpha)((\alpha-1) q+1)^{1 / q}}\left\|_{0}^{c} D_{t}^{\alpha} u\right\|_{L^{p}}
$$

According to (2.8), we can consider $E_{0}^{\alpha, p}$ with respect to the norm

$$
\|u\|_{\alpha, p}=\left\|{ }_{0}^{c} D_{t}^{\alpha} u\right\|_{L^{p}}=\left(\int_{0}^{T}\left|{ }_{0}^{c} D_{t}^{\alpha} u(t)\right|^{p} d t\right)^{1 / p} .
$$

Proposition 2.8 (see [32]). Define $0<\alpha \leq 1$ and $1<p<\infty$. Assume that $\alpha>1 / p$ and the sequence $\left\{u_{k}\right\}$ converges weakly to $u$ in $E_{0}^{\alpha, p}$, that is, $u_{k} \rightarrow u$. Then $u_{k} \rightarrow u$ in $C\left([0, T], \mathbb{R}^{N}\right)$, that is, $\left\|u-u_{k}\right\|_{\infty} \rightarrow 0$, as $k \rightarrow \infty$.

Making use of Property 2.4 and Definition 2.3, for any $u \in A C\left([0, T], \mathbb{R}^{N}\right)$, BVP (1.1) is equivalent to the following problem:

$$
\begin{gathered}
\frac{d}{d t}\left(\frac{1}{2}{ }_{0} D_{t}^{\alpha-1}\left({ }_{0}^{c} D_{t}^{\alpha} u(t)\right)-\frac{1}{2}{ }_{t} D_{T}^{\alpha-1}\left({ }_{t}^{c} D_{T}^{\alpha} u(t)\right)\right)+\nabla F(t, u(t))=0, \quad \text { a.e. } t \in[0, T], \\
u(0)=u(T)=0,
\end{gathered}
$$

where $\alpha=1-\beta / 2 \in(1 / 2,1]$. 
In the following, we will treat BVP (2.10) in the Hilbert space $E^{\alpha}=E_{0}^{\alpha, 2}$ with the corresponding norm $\|u\|_{\alpha}=\|u\|_{\alpha, 2}$. The variational structure of BVP (2.10) on the space $E^{\alpha}$ has been established.

Lemma 2.9 (see [32]). Let $L:[0, T] \times \mathbb{R}^{N} \times \mathbb{R}^{N} \times \mathbb{R}^{N} \rightarrow \mathbb{R}$ be defined by

$$
L(t, x, y, z)=-\frac{1}{2}(y, z)-F(t, x)
$$

where $F:[0, T] \times \mathbb{R}^{N} \rightarrow \mathbb{R}$ satisfies the assumption $(A)$.

If $1 / 2<\alpha \leq 1$, then the functional defined by

$$
\varphi(u)=\int_{0}^{T} L\left(t, u(t),{ }_{0}^{c} D_{t}^{\alpha} u(t),{ }_{t}^{c} D_{T}^{\alpha} u(t)\right) d t
$$

is continuously differentiable on $E^{\alpha}$, and $\forall u, v \in E^{\alpha}$, we have

$$
\begin{aligned}
\left\langle\varphi^{\prime}(u), v\right\rangle= & \int_{0}^{T}\left(D_{x} L\left(t, u(t),{ }_{0}^{c} D_{t}^{\alpha} u(t),{ }_{t}^{c} D_{T}^{\alpha} u(t)\right), v(t)\right) d t \\
& +\int_{0}^{T}\left(D_{y} L\left(t, u(t),{ }_{0}^{c} D_{t}^{\alpha} u(t),{ }_{t}^{c} D_{T}^{\alpha} u(t)\right),{ }_{0}^{c} D_{t}^{\alpha} v(t)\right) d t \\
& +\int_{0}^{T}\left(D_{z} L\left(t, u(t),{ }_{0}^{c} D_{t}^{\alpha} u(t),{ }_{t}^{c} D_{T}^{\alpha} u(t)\right),{ }_{t}^{c} D_{T}^{\alpha} v(t)\right) d t .
\end{aligned}
$$

Definition 2.10 (see [32]). A function $u \in A C\left([0, T], \mathbb{R}^{N}\right)$ is called a solution of BVP (2.10) if

(i) $D^{\alpha}(u(t))$ is derivative for almost every $t \in[0, T]$,

(ii) $u$ satisfies (2.10),

where $D^{\alpha}(u(t)):=(1 / 2){ }_{0} D_{t}^{\alpha-1}\left({ }_{0}^{c} D_{t}^{\alpha} u(t)\right)-(1 / 2){ }_{t} D_{T}^{\alpha-1}\left({ }_{t}^{c} D_{T}^{\alpha} u(t)\right)$.

Lemma 2.11 (see [32]). Let $1 / 2<\alpha \leq 1$ and $\varphi$ be defined by (2.12). If assumption ( $A$ ) is satisfied and $u \in E^{\alpha}$ is a solution of corresponding Euler equation $\varphi^{\prime}(u)=0$, then $u$ is a solution of $B V P$ (2.10) which corresponding to the solution of BVP (1.1).

By Lemma 2.11, it means that the solutions for BVP (1.1) correspond to the critical points of the functional $\varphi$. We need the following estimate and known results for the sequel.

Proposition 2.12 (see [32]). If $1 / 2<\alpha \leq 1$, then for any $u \in E^{\alpha}$, one has

$$
|\cos (\pi \alpha)|\|u\|_{\alpha}^{2} \leq-\int_{0}^{T}\left({ }_{0}^{c} D_{t}^{\alpha} u(t),{ }_{t}^{c} D_{T}^{\alpha} u(t)\right) d t \leq \frac{1}{|\cos (\pi \alpha)|}\|u\|_{\alpha}^{2} .
$$

Lemma 2.13 (see [23]). Let $X$ be a real Banach space, $\Phi: X \rightarrow \mathbb{R}$ is differentiable. One says that $\Phi$ satisfies the (PS) condition if any sequence $\left\{u_{k}\right\}$ in $X$ such that $\left\{\Phi\left(u_{k}\right)\right\}$ is bounded and $\Phi^{\prime}\left(u_{k}\right) \rightarrow 0$ as $k \rightarrow \infty$ contains a convergent subsequence. 
Lemma 2.14 (Mountain Pass theorem [24]). Let $X$ be a real Banach space and $\Phi: X \rightarrow \mathbb{R}$ is differentiable and satisfies the (PS) condition. Suppose that

(i) $\Phi(0)=0$,

(ii) there exist $\rho>0$ and $\sigma>0$ such that $\Phi(z) \geq \sigma$ for all $z \in X$ with $\|z\|=\rho$,

(iii) there exists $z_{1}$ in $X$ with $\left\|z_{1}\right\| \geq \rho$ such that $\Phi\left(z_{1}\right)<\sigma$.

Then $\Phi$ possesses a critical value $c \geq \sigma$. Moreover, $c$ can be characterized as

$$
c=\inf _{g \in \Omega} \max _{z \in g([0,1])} \Phi(z)
$$

where $\bar{\Omega}=\left\{g \in C([0,1], X): g(0)=0, g(1)=z_{1}\right\}$.

Lemma 2.15 (Clark theorem [24]). Let $X$ be a real Banach space, $\Phi \in C^{1}(X, \mathbb{R})$ with $\Phi$ even, bounded below, and satisfying the (PS) condition. Suppose $\Phi(0)=0$, there is a set $K \subset X$ such that $K$ is homeomorphic to $S^{m-1}, m \in \mathbb{N}$, by an odd map, and $\sup _{K} \Phi<0$. Then $\Phi$ possesses at least $m$ distinct pairs of critical points.

\section{Proof of the Theorems}

For $u \in E^{\alpha}$, where

$$
E^{\alpha}:=\left\{u \in L^{2}\left(0, T ; \mathbb{R}^{N}\right):{ }_{0}^{c} D_{t}^{\alpha} u \in L^{2}\left(0, T ; \mathbb{R}^{N}\right)\right\}
$$

is a reflexive Banach space with the norm defined by

$$
\begin{aligned}
\|u\|_{\alpha} & =\left\|{ }_{0}^{c} D_{t}^{\alpha} u\right\|_{L_{2}} \\
\|u\|_{\infty} & :=\max _{t \in[0, T]}|u(t)| .
\end{aligned}
$$

It follows from Lemma 2.9 that the functional $\varphi$ on $E^{\alpha}$ given by

$$
\varphi(u)=\int_{0}^{T}\left[-\frac{1}{2}\left({ }_{0}^{c} D_{t}^{\alpha} u(t),{ }_{t}^{c} D_{T}^{\alpha} u(t)\right)-F(t, u(t))\right] d t
$$

is continuously differentiable on $E^{\alpha}$. Moreover, we have

$$
\begin{aligned}
\left\langle\varphi^{\prime}(u), v\right\rangle= & -\int_{0}^{T} \frac{1}{2}\left[\left({ }_{0}^{c} D_{t}^{\alpha} u(t),{ }_{t}^{c} D_{T}^{\alpha} v(t)\right)+\left({ }_{t}^{c} D_{T}^{\alpha} u(t),{ }_{0}^{c} D_{t}^{\alpha} v(t)\right)\right] d t \\
& -\int_{0}^{T}(\nabla F(t, u(t)), v(t)) d t .
\end{aligned}
$$

Recall that a sequence $\left\{u_{n}\right\} \in E^{\alpha}$ is said to be a (C) sequence of $\varphi$ if $\varphi\left(u_{n}\right)$ is bounded and $\left(1+\left\|u_{n}\right\|_{\alpha}\right)\left\|\varphi\left(u_{n}\right)\right\|_{\alpha} \rightarrow 0$ as $n \rightarrow \infty$. The functional $\varphi$ satisfies condition (C) if every (C) sequence of $\varphi$ has a convergent subsequence. This condition is due to Cerami [21]. 


\subsection{Proof of Theorem 1.1}

We will first establish the following lemma and then give the proof of Theorem 1.1.

Lemma 3.1. Assume (A), (A2), and (A3) hold, then the functional $\varphi$ satisfies condition (C).

Proof of Lemma 3.1. Let $\left\{u_{n}\right\} \subset E^{\alpha}$ be a (C) sequence of $\varphi$, that is, $\varphi\left(u_{n}\right)$ is bounded and $(1+$ $\left.\left\|u_{n}\right\|_{\alpha}\right)\left\|\varphi^{\prime}\left(u_{n}\right)\right\|_{\alpha} \rightarrow 0$ as $n \rightarrow \infty$. Then there exists $M_{0}$ such that

$$
\left|\varphi\left(u_{n}\right)\right| \leq M_{0}, \quad\left(1+\left\|u_{n}\right\|_{\alpha}\right)\left\|\varphi^{\prime}\left(u_{n}\right)\right\|_{\alpha} \leq M_{0},
$$

for all $n \in \mathbb{N}$.

By (A2), there exist positive constants $B_{1}$ and $M_{1}$ such that

$$
F(t, x) \leq B_{1}|x|^{r},
$$

for all $|x| \geq M_{1}$ and a.e. $t \in[0, T]$.

It follows from (A) that

$$
|F(t, x)| \leq \max _{s \in\left[0, M_{1}\right]} a(s) b(t)
$$

for all $|x| \leq M_{1}$ and a.e. $t \in[0, T]$. Therefore, we obtain

$$
F(t, x) \leq B_{1}|x|^{r}+\max _{s \in\left[0, M_{1}\right]} a(s) b(t),
$$

for all $x \in \mathbb{R}^{N}$ and a.e. $t \in[0, T]$.

Combining (2.14) and (3.8), we get

$$
\begin{aligned}
\frac{|\cos (\pi \alpha)|}{2}\left\|u_{n}\right\|_{\alpha}^{2} & \leq \varphi\left(u_{n}\right)+\int_{0}^{T} F\left(t, u_{n}(t)\right) d t \\
& \leq M_{0}+\max _{s \in\left[0, M_{1}\right]} a(s) \int_{0}^{T} b(t) d t+B_{1} \int_{0}^{T}\left|u_{n}(t)\right|^{r} d t .
\end{aligned}
$$

On the other hand, by (A3), there exist $\eta>0$ and $M_{2}>0$ such that

$$
(\nabla F(t, x), x)-2 F(t, x) \geq \eta|x|^{\mu},
$$

for a.e. $t \in[0, T]$ and $|x| \geq M_{2}$.

By (A), we have

$$
|(\nabla F(t, x), x)-2 F(t, x)| \leq\left(2+M_{2}\right) \max _{s \in\left[0, M_{2}\right]} a(s) b(t),
$$

for all $|x| \leq M_{2}$ and a.e. $t \in[0, T]$. 
Abstract and Applied Analysis

Therefore, we obtain

$$
(\nabla F(t, x), x)-2 F(t, x) \geq \eta|x|^{\mu}-\eta M_{2}^{\mu}-\left(2+M_{2}\right) \max _{s \in\left[0, M_{2}\right]} a(s) b(t),
$$

for all $x \in \mathbb{R}^{N}$ and a.e. $t \in[0, T]$.

It follows from (3.5) and (3.12) that

$$
\begin{aligned}
3 M_{0} \geq & 2 \varphi\left(u_{n}\right)-\left\langle\varphi^{\prime}\left(u_{n}\right), u_{n}\right\rangle \\
= & 2 \int_{0}^{T}\left[-\frac{1}{2}\left({ }_{0}^{c} D_{t}^{\alpha} u_{n}(t),{ }_{t}^{c} D_{T}^{\alpha} u_{n}(t)\right)-F\left(t, u_{n}(t)\right)\right] d t \\
& -\int_{0}^{T}\left[-\left({ }_{0}^{c} D_{t}^{\alpha} u_{n}(t),{ }_{t}^{c} D_{T}^{\alpha} u_{n}(t)\right)-\left(\nabla F\left(t, u_{n}(t)\right), u_{n}(t)\right)\right] d t \\
= & \int_{0}^{T}\left[\left(\nabla F\left(t, u_{n}(t)\right), u_{n}(t)\right)-2 F\left(t, u_{n}(t)\right)\right] d t \\
\geq & \eta \int_{0}^{T}\left|u_{n}(t)\right|^{\mu} d t-\left(2+M_{2}\right) \max _{s \in\left[0, M_{2}\right]} a(s) \int_{0}^{T} b(t) d t-\eta M_{2}^{\mu} T,
\end{aligned}
$$

thus, $\int_{0}^{T}\left|u_{n}(t)\right|^{\mu} d t$ is bounded.

If $\mu>r$, then

$$
\int_{0}^{T}\left|u_{n}(t)\right|^{r} d t \leq T^{(\mu-r) / \mu}\left(\int_{0}^{T}\left|u_{n}(t)\right|^{\mu} d t\right)^{r / \mu}
$$

which, combining (3.9), implies that $\left\|u_{n}\right\|_{\alpha}$ is bounded.

If $\mu \leq r$, then

$$
\int_{0}^{T}\left|u_{n}(t)\right|^{r} d t \leq\left\|u_{n}\right\|_{\infty}^{r-\mu} \int_{0}^{T}\left|u_{n}(t)\right|^{\mu} d t \leq C_{1}^{r-\mu}\left\|u_{n}\right\|_{\alpha}^{r-\mu} \int_{0}^{T}\left|u_{n}(t)\right|^{\mu} d t,
$$

where

$$
C_{1}:=\frac{T^{\alpha-1 / 2}}{\Gamma(\alpha)(2 \alpha-1)^{1 / 2}}
$$

by (2.8).

Since $\mu>r-2$, it follows from (3.9) that $\left\|u_{n}\right\|_{\alpha}$ is bounded too. Thus $\left\|u_{n}\right\|_{\alpha}$ is bounded in $E^{\alpha}$. that

By Proposition 2.8, the sequence $\left\{u_{n}\right\}$ has a subsequence, also denoted by $\left\{u_{n}\right\}$, such

$$
u_{n} \rightarrow u \quad \text { weakly in } E^{\alpha}, \quad u_{n} \longrightarrow u \text { strongly in } C\left([0, T], \mathbb{R}^{N}\right)
$$


Then we obtain $u_{n} \rightarrow u$ in $E^{\alpha}$ by use of the same argument of Theorem 5.2 in [32]. The proof of Lemma 3.1 is completed.

Proof of Theorem 1.1. By (A1), there exist $\epsilon_{1} \in(0,|\cos (\pi \alpha)|)$ and $\delta>0$ such that

$$
F(t, x) \leq\left(|\cos (\pi \alpha)|-\epsilon_{1}\right) \frac{\Gamma^{2}(\alpha+1)}{2 T^{2 \alpha}}|x|^{2},
$$

for a.e. $t \in[0, T]$ and $x \in \mathbb{R}^{N}$ with $|x| \leq \delta$.

Let

$$
\rho=\frac{\Gamma(\alpha)(2(\alpha-1)+1)^{1 / 2}}{T^{\alpha-1 / 2}} \delta, \quad \sigma=\frac{\epsilon_{1} \rho^{2}}{2}>0 .
$$

Then it follows from (2.8) that

$$
\|u\|_{\infty} \leq \frac{T^{\alpha-1 / 2}}{\Gamma(\alpha)(2(\alpha-1)+1)^{1 / 2}}\|u\|_{\alpha}=\delta
$$

for all $u \in E^{\alpha}$ with $\|u\|_{\alpha}=\rho$.

Therefore, we have

$$
\begin{aligned}
\varphi(u) & =\int_{0}^{T}\left[-\frac{1}{2}\left({ }_{0}^{c} D_{t}^{\alpha} u(t),{ }_{t}^{c} D_{T}^{\alpha} u(t)\right)-F(t, u(t))\right] d t \\
& \geq \frac{|\cos (\pi \alpha)|}{2}\|u\|_{\alpha}^{2}-\left(|\cos (\pi \alpha)|-\epsilon_{1}\right) \frac{\Gamma^{2}(\alpha+1)}{2 T^{2 \alpha}} \int_{0}^{T}|u(t)|^{2} d t \\
& \geq \frac{|\cos (\pi \alpha)|}{2}\|u\|_{\alpha}^{2}-\frac{|\cos (\pi \alpha)|-\epsilon_{1}}{2}\|u\|_{\alpha}^{2} \\
& =\frac{\epsilon_{1}}{2}\|u\|_{\alpha}^{2} \\
& =\sigma,
\end{aligned}
$$

for all $u \in E^{\alpha}$ with $\|u\|_{\alpha}=\rho$. This implies that (ii) in Lemma 2.14 is satisfied.

It is obvious from the definition of $\varphi$ and (A1) that $\varphi(0)=0$, and therefore, it suffices to show that $\varphi$ satisfies (iii) in Lemma 2.14.

By (A1), there exist $\epsilon_{2}>0$ and $M_{3}>0$ such that

$$
F(t, x)>\left(\frac{\pi^{2}}{|\cos (\pi \alpha)| \Gamma^{2}(2-\alpha) T^{2 \alpha}(3-2 \alpha)}+\epsilon_{2}\right)|x|^{2},
$$

for all $|x| \geq M_{3}$ and a.e. $t \in[0, T]$. 
Abstract and Applied Analysis

It follows from (A) that

$$
|F(t, x)| \leq \max _{s \in\left[0, M_{3}\right]} a(s) b(t),
$$

for all $|x| \leq M_{3}$ and a.e. $t \in[0, T]$.

Therefore, we obtain

$$
F(t, x) \geq\left(\frac{\pi^{2}}{|\cos (\pi \alpha)| \Gamma^{2}(2-\alpha) T^{2 \alpha}(3-2 \alpha)}+\epsilon_{2}\right)\left(|x|^{2}-M_{3}^{2}\right)-\max _{s \in\left[0, M_{3}\right]} a(s) b(t),
$$

for all $x \in \mathbb{R}^{N}$ and a.e. $t \in[0, T]$.

Choosing $u_{0}=((T / \pi) \sin (\pi t / T), 0, \ldots, 0) \in E^{\alpha}$, then

$$
\left\|u_{0}\right\|_{L_{2}}^{2}=\frac{T^{3}}{2 \pi^{2}}, \quad\left\|u_{0}\right\|_{\alpha}^{2} \leq \frac{T^{3-2 \alpha}}{\Gamma^{2}(2-\alpha)(3-2 \alpha)} .
$$

For $\varsigma>0$ and noting that (3.24) and (3.25), we have

$$
\begin{aligned}
\varphi\left(\varsigma u_{0}\right)= & \int_{0}^{T}\left[-\frac{1}{2}\left({ }_{0}^{c} D_{t}^{\alpha} s u_{0}(t),{ }_{t}^{c} D_{T}^{\alpha} s u_{0}(t)\right)-F\left(t, \varsigma u_{0}(t)\right)\right] d t \\
\leq & \frac{\varsigma^{2}}{2|\cos (\pi \alpha)|}\left\|u_{0}\right\|_{\alpha}^{2}-\left(\frac{\varsigma^{2} \pi^{2}}{|\cos (\pi \alpha)| T^{2 \alpha} \Gamma^{2}(2-\alpha)(3-2 \alpha)}+\varsigma^{2} \epsilon_{2}\right) \int_{0}^{T}\left|u_{0}(t)\right|^{2} d t+C_{2} \\
\leq & \frac{\varsigma^{2}}{2|\cos (\pi \alpha)|} \cdot \frac{T^{3-2 \alpha}}{\Gamma^{2}(2-\alpha)(3-2 \alpha)}-\frac{\varsigma^{2} \pi^{2}}{|\cos (\pi \alpha)| T^{2 \alpha} \Gamma^{2}(2-\alpha)(3-2 \alpha)} \cdot \frac{T^{3}}{2 \pi^{2}} \\
& -\frac{\varsigma^{2} \epsilon_{2} T^{3}}{2 \pi^{2}}+C_{2} \\
& \longrightarrow-\infty
\end{aligned}
$$

as $\varsigma \rightarrow \infty$, where $C_{2}$ is a positive constant. Then there exists a sufficiently large $\varsigma_{0}$ such that $\varphi\left(\varsigma_{0} u_{0}\right) \leq 0$. Hence (iii) holds.

Finally, noting that $\varphi(0)=0$ while for critical point $u, \varphi(u) \geq \sigma>0$. Hence $u$ is a nontrivial solution of BVP (1.1), and this completes the proof.

\subsection{Proof of Theorem 1.2}

The following lemmata are needed in the proof of Theorem 1.2. 
Lemma 3.2. Assume (A5), then for any $\varepsilon>0$, there exists a subset $E_{\varepsilon} \subset[0, T]$ with meas $([0, T] \backslash$ $\left.E_{\varepsilon}\right)<\varepsilon$ such that

$$
\lim _{|x| \rightarrow \infty}[(\nabla F(t, x), x)-2 F(t, x)]=+\infty
$$

uniformly for $t \in E_{\varepsilon}$.

Proof of Lemma 3.2. The proof is similar to that of Lemma 2 in [29] and is omitted.

Lemma 3.3. Assume (A), (A2'), (A4), and (A5), then the functional $\varphi$ satisfies condition (C).

Proof of Lemma 3.3. Suppose that $\left\{u_{n}\right\} \subset E^{\alpha}$ is a (C) sequence of $\varphi$, that is, $\varphi\left(u_{n}\right)$ is bounded and $\left(1+\left\|u_{n}\right\|_{\alpha}\right)\left\|\varphi^{\prime}\left(u_{n}\right)\right\|_{\alpha} \rightarrow 0$ as $n \rightarrow \infty$. Then we have

$$
\liminf _{n \rightarrow \infty}\left[\left\langle\varphi^{\prime}\left(u_{n}\right), u_{n}\right\rangle-2 \varphi\left(u_{n}\right)\right]>-\infty,
$$

which implies that

$$
\limsup _{n \rightarrow \infty} \int_{0}^{T}\left[\left(\nabla F\left(t, u_{n}\right), u_{n}\right)-2 F\left(t, u_{n}\right)\right] d t<+\infty
$$

We only need to show that $\left\{u_{n}\right\}$ is bounded in $E^{\alpha}$. If $\left\{u_{n}\right\}$ is unbounded, we may assume, without loss of generality, that $\left\|u_{n}\right\|_{\alpha} \rightarrow \infty$ as $n \rightarrow \infty$. Put $z_{n}=u_{n} /\left\|u_{n}\right\|_{\alpha}$, we then have $\left\|z_{n}\right\|_{\alpha}=1$. Going to a sequence if necessary, we assume that $z_{n} \rightarrow z$ weakly in $E^{\alpha}$, $z_{n} \rightarrow z$ strongly in $C\left([0, T], \mathbb{R}^{N}\right)$ and $L^{2}\left(0, T ; \mathbb{R}^{N}\right)$.

By (A2), it follows that there exist constants $B_{2}>0$ and $M_{4}>0$ such that

$$
F(t, x) \leq B_{2}|x|^{2}
$$

for all $|x| \geq M_{4}$ and a.e. $t \in[0, T]$.

By assumption (A), it follows that

$$
|F(t, x)| \leq \max _{s \in\left[0, M_{4}\right]} a(s) b(t),
$$

for all $|x| \leq M_{4}$ and a.e. $t \in[0, T]$. Therefore, we obtain

$$
F(t, x) \leq B_{2}|x|^{2}+\max _{s \in\left[0, M_{4}\right]} a(s) b(t)
$$

for all $x \in \mathbb{R}^{N}$ and a.e. $t \in[0, T]$. Therefore, we have

$$
\begin{aligned}
\varphi(u) & =\int_{0}^{T}\left[-\frac{1}{2}\left({ }_{0}^{c} D_{t}^{\alpha} u(t),{ }_{t}^{c} D_{T}^{\alpha} u(t)\right)-F(t, u(t))\right] d t \\
& \geq \frac{|\cos (\pi \alpha)|}{2}\|u\|_{\alpha}^{2}-B_{2} \int_{0}^{T}|u|^{2} d t-\max _{s \in\left[0, M_{4}\right]} a(s) \int_{0}^{T} b(t) d t
\end{aligned}
$$


from which, it follows that

$$
\frac{\varphi\left(u_{n}\right)}{\left\|u_{n}\right\|_{\alpha}^{2}} \geq \frac{|\cos (\pi \alpha)|}{2}-B_{2}\left\|z_{n}\right\|_{L_{2}}^{2}-\frac{1}{\left\|u_{n}\right\|_{\alpha}^{2}} \max _{s \in\left[0, M_{4}\right]} a(s) \int_{0}^{T} b(t) d t
$$

Passing to the limit in the last inequality, we get

$$
\frac{|\cos (\pi \alpha)|}{2}-B_{2}\|z\|_{L_{2}}^{2} \leq 0
$$

which yields $z \neq 0$. Therefore, there exists a subset $E \subset[0, T]$ with meas $(E)>0$ such that $z(t) \neq 0$ on $E$.

By virtue of Lemma 3.2, for $\varepsilon=(1 / 2)$ meas $(E)>0$, we can choose a subset $E_{\varepsilon} \subset[0, T]$ with meas $\left([0, T] \backslash E_{\varepsilon}\right)<\varepsilon$ such that

$$
\lim _{|x| \rightarrow \infty}[(\nabla F(t, x), x)-2 F(t, x)]=+\infty
$$

uniformly for $t \in E_{\varepsilon}$.

We assert that meas $\left(E \cap E_{\varepsilon}\right)>0$. If not, meas $\left(E \cap E_{\varepsilon}\right)=0$.

Since $E=\left(E \cap E_{\varepsilon}\right) \cup\left(E \backslash E_{\varepsilon}\right)$, it follows that

$$
\begin{aligned}
0<\operatorname{meas}(E) & =\operatorname{meas}\left(E \cap E_{\varepsilon}\right)+\operatorname{meas}\left(E \backslash E_{\varepsilon}\right) \\
& \leq \operatorname{meas}\left([0, T] \backslash E_{\varepsilon}\right) \\
& <\varepsilon=\frac{1}{2} \operatorname{meas}(E),
\end{aligned}
$$

which leads to a contradiction and establishes the assertion.

By (A4), we obtain thye following:

$$
\begin{aligned}
& \int_{0}^{T}\left[\left(\nabla F\left(t, u_{n}\right), u_{n}\right)-2 F\left(t, u_{n}\right)\right] d t \\
& \quad=\int_{E \cap E_{\varepsilon}}\left[\left(\nabla F\left(t, u_{n}\right), u_{n}\right)-2 F\left(t, u_{n}\right)\right] d t+\int_{[0, T] \backslash\left(E \cap E_{\varepsilon}\right)}\left[\left(\nabla F\left(t, u_{n}\right), u_{n}\right)-2 F\left(t, u_{n}\right)\right] d t \\
& \quad \geq \int_{E \cap E_{\varepsilon}}\left[\left(\nabla F\left(t, u_{n}\right), u_{n}\right)-2 F\left(t, u_{n}\right)\right] d t-\int_{0}^{T}|\tau(t)| d t .
\end{aligned}
$$

By (3.36), (3.38), and Fatou's lemma, it follows that

$$
\lim _{n \rightarrow \infty} \int_{0}^{T}\left[\left(\nabla F\left(t, u_{n}\right), u_{n}\right)-2 F\left(t, u_{n}\right)\right] d t=+\infty,
$$


which contradicts (3.29). This contradiction shows that $\left\|u_{n}\right\|_{\alpha}$ is bounded in $E^{\alpha}$, and this completes the proof.

By virtue of Lemmas 3.2 and 3.3, the rest of the proof is similar to Theorem 1.1. Theorem 1.3 can be proved similarly.

\subsection{Proof of Theorem 1.4}

The proof of Theorem 1.4 is divided into a sequence of lemma.

Lemma 3.4. The functional $\varphi$ is bounded below on $E^{\alpha}$.

Proof of Lemma 3.4. By (2.8) and (2.14), for every $u \in E^{\alpha}$, we have

$$
\begin{aligned}
\varphi(u) & =-\int_{0}^{T} \frac{1}{2}\left({ }_{0}^{c} D_{t}^{\alpha} u(t),{ }_{t}^{c} D_{T}^{\alpha} u(t)\right) d t-\int_{0}^{T} F(t, u(t)) d t \\
& =-\int_{0}^{T} \frac{1}{2}\left({ }_{0}^{c} D_{t}^{\alpha} u(t),{ }_{t}^{c} D_{T}^{\alpha} u(t)\right) d t-\int_{0}^{T} a(t)|u(t)|^{\gamma} d t \\
& \geq \frac{|\cos (\pi \alpha)|}{2}\|u\|_{\alpha}^{2}-a_{0}\|u\|_{\infty}^{\gamma} T \\
& \geq \frac{|\cos (\pi \alpha)|}{2}\|u\|_{\alpha}^{2}-a_{0} T C_{1}^{\gamma}\|u\|_{\alpha,}^{\gamma}
\end{aligned}
$$

where $a_{0}=\operatorname{ess} \sup \{a(t): t \in[0, T]\}$. The proof of Lemma 3.4 is complete.

Lemma 3.5. The functional $\varphi$ satisfies the (PS) condition.

Proof of Lemma 3.5. Let $\left\{u_{n}\right\}$ be a Palais-Smale sequence in $E^{\alpha}$, that is,

$$
\varphi\left(u_{n}\right) \text { is bounded and } \varphi^{\prime}\left(u_{n}\right) \longrightarrow 0 \text { as } n \longrightarrow+\infty \text {. }
$$

Suppose that $\left\{u_{n}\right\}$ is unbounded in $E^{\alpha}$, that is, $\left\|u_{n}\right\|_{\alpha} \rightarrow+\infty$ as $n \rightarrow+\infty$. Since

$$
\left\langle\varphi^{\prime}\left(u_{n}\right), u_{n}\right\rangle-\gamma \varphi\left(u_{n}\right)=\left(-1+\frac{\gamma}{2}\right) \int_{0}^{T}\left({ }_{0}^{c} D_{t}^{\alpha} u_{n}(t){ }_{t}^{c} D_{T}^{\alpha} u_{n}(t)\right) d t
$$

However, from (3.42), we have

$$
-\gamma \varphi\left(u_{n}\right) \geq\left(1-\frac{\gamma}{2}\right)|\cos (\pi \alpha)|\left\|u_{n}\right\|_{\alpha}^{2}-\left\|\varphi^{\prime}\left(u_{n}\right)\right\|\left\|u_{n}\right\|_{\alpha^{\prime}}
$$

thus $\left\|u_{n}\right\|_{\alpha}$ is a bounded sequence in $E^{\alpha}$. Since $E^{\alpha}$ is a reflexive space, going, if necessary, to a subsequence, we can assume that $u_{n} \rightarrow u$ in $E^{\alpha}$, thus we have

$$
\begin{aligned}
\left\langle\varphi^{\prime}\left(u_{n}\right)-\varphi^{\prime}(u), u_{n}-u\right\rangle & =\left\langle\varphi^{\prime}\left(u_{n}\right), u_{n}-u\right\rangle-\left\langle\varphi^{\prime}(u), u_{n}-u\right\rangle \\
& \leq\left\|\varphi^{\prime}\left(u_{n}\right)\right\|_{\alpha}\left\|u_{n}-u\right\|_{\alpha}-\left\langle\varphi^{\prime}(u), u_{n}-u\right\rangle \longrightarrow 0,
\end{aligned}
$$


as $n \rightarrow \infty$. Moreover, according to (2.8) and Proposition 2.8, we have that $\left\{u_{n}\right\}$ is bounded in $C\left([0, T], \mathbb{R}^{N}\right)$ and $\left\|u_{n}-u\right\|_{\infty} \rightarrow 0$ as $n \rightarrow \infty$.

Noting that

$$
\begin{aligned}
\left\langle\varphi^{\prime}\left(u_{n}\right)\right. & \left.-\varphi^{\prime}(u), u_{n}-u\right\rangle \\
= & -\int_{0}^{T}\left({ }_{0}^{c} D_{t}^{\alpha}\left(u_{n}(t)-u(t)\right),{ }_{0}^{c} D_{T}^{\alpha}\left(u_{n}(t)-u(t)\right)\right) d t \\
& -\int_{0}^{T}\left(\nabla F\left(t, u_{n}(t)\right)-\nabla F(t, u(t)), u_{n}(t)-u(t)\right) d t \\
\geq & |\cos (\pi \alpha)|\left\|u_{n}-u\right\|_{\alpha}^{2}-\left|\int_{0}^{T}\left(\nabla F\left(t, u_{n}(t)\right)-\nabla F(t, u(t))\right) d t\right|\left\|u_{n}-u\right\|_{\infty} .
\end{aligned}
$$

Combining (3.44) and (3.45), it is easy to verify that $\left\|u_{n}-u\right\|_{\alpha} \rightarrow 0$ as $n \rightarrow \infty$, and hence that $u_{n} \rightarrow u$ in $E^{\alpha}$. Thus, $\left\{u_{n}\right\}$ admits a convergent subsequence. The proof of Lemma 3.5 is complete.

Lemma 3.6. For any $m \in \mathbb{N}$, there exists a set $K \subset E^{\alpha}$ which is homeomorphic to $S^{m-1}$ by an odd map, and $\sup _{k} \varphi<0$.

Proof of Lemma 3.6. For every $m \in \mathbb{N}$, define

$$
\begin{gathered}
u_{i}(t)=\left(\sin \frac{i \pi t}{T}, 0, \ldots, 0\right), \quad i=1,2, \ldots, m, \\
E_{m}=\operatorname{span}\left\{u_{1}, \ldots, u_{m}\right\}, \\
K_{m, \beta}=\left\{u \in E_{m}:\|u\|_{\alpha}=\beta\right\},
\end{gathered}
$$

where $\beta$ is a positive number to be chosen later.

For any $u \in E_{m}$, there exist $\lambda_{i} \in \mathbb{R}, i=1,2, \ldots, m$, such that

$$
\begin{aligned}
u & =\sum_{i=1}^{m} \lambda_{i} u_{i}(t) \\
\|u\|_{\alpha}^{2} & =\int_{0}^{T}\left|{ }_{0}^{c} D_{t}^{\alpha} u(t)\right|^{2} d t \\
& =\int_{0}^{T}\left({ }_{0}^{c} D_{t}^{\alpha} u(t),{ }_{0}^{c} D_{t}^{\alpha} u(t)\right) d t \\
& =\int_{0}^{T}\left(\lambda_{1}{ }_{0}^{c} D_{t}^{\alpha} u_{1}(t)+\cdots+\lambda_{m}{ }_{0}^{c} D_{t}^{\alpha} u_{m}(t), \lambda_{1}{ }_{0}^{c} D_{t}^{\alpha} u_{1}(t)+\cdots+\lambda_{m}{ }_{0}^{c} D_{t}^{\alpha} u_{m}(t)\right) d t \\
& =\sum_{i=1}^{m} \sum_{j=1}^{m} a_{i j} \lambda_{i} \lambda_{j}=F\left(\lambda_{1}, \ldots, \lambda_{m}\right),
\end{aligned}
$$

where $a_{i j}=\int_{0}^{T}\left({ }_{0}^{c} D_{t}^{\alpha} u_{i}(t),{ }_{0}^{c} D_{t}^{\alpha} u_{j}(t)\right) d t$ and $F\left(\lambda_{1}, \ldots, \lambda_{m}\right)$ is a real quadratic form. 
Since

$$
\begin{gathered}
F\left(\lambda_{1}, \ldots, \lambda_{m}\right)=\left\|\sum_{i=1}^{m} \lambda_{i} u_{i}(t)\right\|_{\alpha}^{2} \geq 0, \quad \forall\left(\lambda_{1}, \ldots, \lambda_{m}\right)^{T} \in \mathbb{R}^{m}, \\
F\left(\lambda_{1}, \ldots, \lambda_{m}\right)=0 \Longleftrightarrow \sum_{i=1}^{m} \lambda_{i} u_{i}(t) \equiv 0 \\
\Longleftrightarrow \lambda_{1}=\lambda_{2}=\cdots=\lambda_{m}=0 .
\end{gathered}
$$

So, $F\left(\lambda_{1}, \ldots, \lambda_{m}\right)$ is a real positive definite quadratic form. Then there exist an invertible matrix $C \in \mathbb{R}^{m \times m}$ and $\mu_{i} \in \mathbb{R}, i=1,2, \ldots, m$, such that

$$
\begin{gathered}
\left(\lambda_{1}, \lambda_{2}, \ldots, \lambda_{m}\right)^{T}=C\left(\mu_{1}, \mu_{2}, \ldots, \mu_{m}\right)^{T}, \\
F\left(\lambda_{1}, \ldots, \lambda_{m}\right)=\sum_{i=1}^{m} \mu_{i}^{2} .
\end{gathered}
$$

It is easy to prove that the odd mapping $\Psi: K_{m, \beta} \rightarrow S^{m-1}$ defined by

$$
\Psi(u)=\beta^{-1}\left(\mu_{1}, \ldots, \mu_{m}\right)
$$

is a homeomorphism between $K_{m, \beta}$ and $S^{m-1}$.

Since $E_{m} \subset E^{\alpha}$ is a finite dimensional space, there exists $\varepsilon(m)>0$ such that

$$
\operatorname{meas}\left\{t \in[0, T]: a(t)|u(t)|^{\gamma} \geq \varepsilon\|u\|_{\alpha}^{\gamma}\right\} \geq \varepsilon, \quad \forall u \in E_{m} \backslash\{0\} .
$$

Otherwise, for any positive integer $n$, there exists $u_{n} \in E_{m} \backslash\{0\}$ such that

$$
\text { meas }\left\{t \in[0, T]: a(t)\left|u_{n}(t)\right|^{\gamma} \geq \frac{1}{n}\left\|u_{n}\right\|_{\alpha}^{\gamma}\right\}<\frac{1}{n} \text {. }
$$

Set $v_{n}(t):=u_{n}(t) /\left\|u_{n}\right\|_{\alpha} \in E_{m} \backslash\{0\}$, then $\left\|v_{n}\right\|_{\alpha}=1$ for all $n \in \mathbb{N}$ and

$$
\operatorname{meas}\left\{t \in[0, T]: a(t)\left|v_{n}(t)\right|^{\gamma} \geq \frac{1}{n}\right\}<\frac{1}{n} \text {. }
$$

Since $\operatorname{dim} E_{m}<\infty$, it follows from the compactness of the unit sphere of $E_{m}$ that there exists a subsequence, denoted also by $\left\{v_{n}\right\}$, such that $\left\{v_{n}\right\}$ converges to some $v_{0}$ in $E_{m}$. It is obvious that $\left\|v_{0}\right\|_{\alpha}=1$.

By the equivalence of the norms on the finite dimensional space, we have $v_{n} \rightarrow v_{0}$ in $L^{2}\left(0, T ; \mathbb{R}^{N}\right)$, that is,

$$
\int_{0}^{T}\left|v_{n}-v_{0}\right|^{2} d t \longrightarrow 0 \text { as } n \longrightarrow \infty
$$


By (3.54) and Hölder inequality, we have

$$
\begin{aligned}
\int_{0}^{T} a(t)\left|v_{n}-v_{0}\right|^{\gamma} d t & \leq\left(\int_{0}^{T} a(t)^{2 /(2-\gamma)} d t\right)^{(2-\gamma) / 2}\left(\int_{0}^{T}\left|v_{n}-v_{0}\right|^{2} d t\right)^{\gamma / 2} \\
& =\|a\|_{(2-\gamma) / 2}\left(\int_{0}^{T}\left|v_{n}-v_{0}\right|^{2} d t\right)^{\gamma / 2} \longrightarrow 0, \quad \text { as } n \longrightarrow \infty
\end{aligned}
$$

Thus, there exist $\xi_{1}, \xi_{2}>0$ such that

$$
\operatorname{meas}\left\{t \in[0, T]: a(t)\left|v_{0}(t)\right|^{\gamma} \geq \xi_{1}\right\} \geq \xi_{2} \text {. }
$$

In fact, if not, we have

$$
\text { meas }\left\{t \in[0, T]: a(t)\left|v_{0}(t)\right|^{\gamma} \geq \frac{1}{n}\right\}=0,
$$

for all positive integer $n$.

It implies that

$$
0 \leq \int_{0}^{T} a(t)\left|v_{0}\right|^{\gamma+2} d t<\frac{T}{n}\left\|v_{0}\right\|_{\infty}^{2} \leq \frac{C_{1}^{2} T}{n}\left\|v_{0}\right\|_{\alpha}^{2} \longrightarrow 0
$$

as $n \rightarrow \infty$. Hence $v_{0}=0$ which contradicts that $\left\|v_{0}\right\|_{\alpha}=1$. Therefore, (3.56) holds.

Now let

$$
\Omega_{0}=\left\{t \in[0, T]: a(t)\left|v_{0}(t)\right|^{\gamma} \geq \xi_{1}\right\}, \quad \Omega_{n}=\left\{t \in[0, T]: a(t)\left|v_{n}(t)\right|^{\gamma}<\frac{1}{n}\right\},
$$

and $\Omega_{n}^{c}=[0, T] \backslash \Omega_{n}=\left\{t \in[0, T]: a(t)\left|v_{n}(t)\right|^{r} \geq 1 / n\right\}$.

By (3.53) and (3.56), we have

$$
\begin{aligned}
\operatorname{meas}\left(\Omega_{n} \cap \Omega_{0}\right) & =\operatorname{meas}\left(\Omega_{0} \backslash\left(\Omega_{n}^{c} \cap \Omega_{0}\right)\right) \\
& \geq \operatorname{meas}\left(\Omega_{0}\right)-\operatorname{meas}\left(\Omega_{n}^{c} \cap \Omega_{0}\right) \\
& \geq \xi_{2}-\frac{1}{n^{\prime}}
\end{aligned}
$$

for all positive integer $n$. Let $n$ be large enough such that

$$
\xi_{2}-\frac{1}{n} \geq \frac{1}{2} \xi_{2}, \quad \frac{1}{2^{\gamma-1}} \xi_{1}-\frac{1}{n} \geq \frac{1}{2^{\gamma}} \xi_{1}
$$


then we have

$$
\begin{aligned}
\int_{0}^{T} a(t)\left|v_{n}-v_{0}\right|^{\gamma} d t & \geq \int_{\Omega_{n} \cap \Omega_{0}} a(t)\left|v_{n}-v_{0}\right|^{\gamma} d t \\
& \geq \frac{1}{2^{\gamma-1}} \int_{\Omega_{n} \cap \Omega_{0}} a(t)\left|v_{0}\right|^{\gamma} d t-\int_{\Omega_{n} \cap \Omega_{0}} a(t)\left|v_{n}\right|^{\gamma} d t \\
& \geq\left(\frac{1}{2^{\gamma-1}} \xi_{1}-\frac{1}{n}\right) \operatorname{meas}\left(\Omega_{n} \cap \Omega_{0}\right) \\
& \geq \frac{\xi_{1}}{2^{\gamma}} \cdot \frac{\xi_{2}}{2}=\frac{\xi_{1} \xi_{2}}{2^{\gamma+1}}>0
\end{aligned}
$$

for all large $n$, which is a contradiction to (3.55). Therefore, (3.51) holds.

For any $u \in K_{m, \beta}$, we have

$$
\begin{aligned}
\varphi(u) & =-\int_{0}^{T} \frac{1}{2}\left({ }_{0}^{c} D_{t}^{\alpha} u(t){ }_{{ }_{t}}^{c} D_{T}^{\alpha} u(t)\right) d t-\int_{0}^{T} F(t, u(t)) d t \\
& \leq \frac{1}{2|\cos (\pi \alpha)|}\|u\|_{\alpha}^{2}-\int_{0}^{T} a(t)|u(t)|^{\gamma} d t \\
& \leq \frac{1}{2|\cos (\pi \alpha)|}\|u\|_{\alpha}^{2}-\varepsilon\|u\|_{\alpha}^{\gamma} \operatorname{meas}\left(\Omega_{u}\right) \\
& \leq \frac{1}{2|\cos (\pi \alpha)|}\|u\|_{\alpha}^{2}-\varepsilon^{2}\|u\|_{\alpha}^{\gamma},
\end{aligned}
$$

by (3.51), where $\Omega_{u}:=\left\{t \in[0, T]: a(t)|u(t)|^{\gamma} \geq \varepsilon\|u\|_{\alpha}^{\gamma}\right\}$.

Choosing $\beta=\left(|\cos (\pi \alpha)| \varepsilon^{2}\right)^{1 /(2-\gamma)}$, we conclude $\sup _{K_{m, \beta}} \varphi<-\varepsilon^{2} \beta^{\gamma} / 2<0$ which completes the proof.

Now from the assertion of Lemma 2.15, we know that $\varphi$ has at least $m$ distinct pairs of critical points for every $m \in \mathbb{N}$, therefore, BVP (1.1) possesses infinitely many solutions on $E^{\alpha}$. The proof of Theorem 1.4 is completed.

\section{Examples}

In this section, we give some examples to illustrate our results.

Example 4.1. In BVP (1.1), let

$$
F(t, x)=\ln \left(1+2|x|^{2}\right)|x|^{2} .
$$

These show that all conditions of Theorem 1.1 are satisfied, where

$$
r=2.5, \quad \mu=2 .
$$

By Theorem 1.1, BVP (1.1) has at least one solution $u \in E^{\alpha}$. 
Example 4.2. In BVP (1.1), let $T=2 \pi$ and $F(t, x)=\kappa f(x)(2+\sin t) \arctan |x|^{2}$, where $\kappa>0$ and $f(x)$ will be specified below.

Let $f(x)=|x|^{2}+\ln \left(1+|x|^{2}\right)$. Noting that $0 \leq \ln \left(1+|x|^{2}\right) \leq|x|^{2}$, we see that (A) and $\left(\mathrm{A} 2^{\prime}\right)$ hold. It is also easy to see that (A1) holds for

$$
\kappa>\frac{(2 \pi)^{1-2 \alpha}}{|\cos (\pi \alpha)| \Gamma^{2}(2-\alpha)(3-2 \alpha)} .
$$

Furthermore, we have

$$
(\nabla f(x), x)-2 f(x)=\frac{2|x|^{2}}{1+|x|^{2}}-2 \ln \left(1+|x|^{2}\right) \longrightarrow-\infty,
$$

as $|x| \rightarrow+\infty$. Therefore, we have

$$
\begin{aligned}
(\nabla F(t, x), x)-2 F(t, x) & =\kappa \frac{2|x|^{2}}{1+|x|^{4}} f(x)(2+\sin t)+\kappa[(\nabla f(x), x)-2 f(x)](2+\sin t) \arctan |x|^{2} \\
& \longrightarrow-\infty
\end{aligned}
$$

uniformly for all $t \in[0,2 \pi]$ as $|x| \rightarrow+\infty$. Thus $\left(\mathrm{A} 4^{\prime}\right)$ and $\left(\mathrm{A} 5^{\prime}\right)$ hold. By virtue of Theorem 1.3, we conclude that BVP (1.1) has at least one solution on $E^{\alpha}$.

If $f(x)=|x|^{2}-\ln \left(1+|x|^{2}\right)$, then exactly the same conclusions as above hold true by Theorem 1.2.

Example 4.3. In BVP (1.1), let $F(t, x)=a(t)|x|^{3 / 2}$ where

$$
a(t)= \begin{cases}T, & t=0 \\ 2 t, & 0<t \leq \frac{T}{2} \\ -2(t-T), & \frac{T}{2}<t<T \\ T, & t=T .\end{cases}
$$

By Theorem 1.4, BVP (1.1) has infinite solutions on $E^{\alpha}$.

\section{Acknowledgment}

This paper is partially supported by the NNSF (no. 11171351) of China.

\section{References}

[1] A. A. Kilbas, H. M. Srivastava, and J. J. Trujillo, Theory and Applications of Fractional Differential Equations, vol. 204 of North-Holland Mathematics Studies, Elsevier Science B. V., Amsterdam, The Netherlands, 2006. 
[2] K. S. Miller and B. Ross, An Introduction to the Fractional Calculus and Fractional Differential Equations, A Wiley-Interscience Publication, John Wiley \& Sons, New York, NY, USA, 1993.

[3] I. Podlubny, Fractional Differential Equations, vol. 198 of Mathematics in Science and Engineering, Academic Press, San Diego, Calif, USA, 1999.

[4] S. G. Samko, A. A. Kilbas, and O. I. Marichev, Fractional Integrals and Derivatives: Theory and Applications, Gordon and Breach, Longhorne, Pa, USA, 1993.

[5] M. Benchohra, S. Hamani, and S. K. Ntouyas, "Boundary value problems for differential equations with fractional order and nonlocal conditions," Nonlinear Analysis: Theory, Methods E Applications A, vol. 71, no. 7-8, pp. 2391-2396, 2009.

[6] R. P. Agarwal, M. Benchohra, and S. Hamani, "A survey on existence results for boundary value problems of nonlinear fractional differential equations and inclusions," Acta Applicandae Mathematicae, vol. 109, no. 3, pp. 973-1033, 2010.

[7] V. Lakshmikantham and A. S. Vatsala, "Basic theory of fractional differential equations," Nonlinear Analysis: Theory, Methods \& Applications A, vol. 69, no. 8, pp. 2677-2682, 2008.

[8] J. Vasundhara Devi and V. Lakshmikantham, "Nonsmooth analysis and fractional differential equations," Nonlinear Analysis: Theory, Methods E Applications A, vol. 70, no. 12, pp. 4151-4157, 2009.

[9] B. Ahmad, "Existence of solutions for irregular boundary value problems of nonlinear fractional differential equations," Applied Mathematics Letters, vol. 23, no. 4, pp. 390-394, 2010.

[10] Y. Zhou, F. Jiao, and J. Li, "Existence and uniqueness for fractional neutral differential equations with infinite delay," Nonlinear Analysis: Theory, Methods \& Applications A, vol. 71, no. 7-8, pp. 3249-3256, 2009.

[11] J. Rong Wang and Y. Zhou, "A class of fractional evolution equations and optimal controls," Nonlinear Analysis. Real World Applications, vol. 12, no. 1, pp. 262-272, 2011.

[12] Z. Bai and H. Lü, "Positive solutions for boundary value problem of nonlinear fractional differential equation," Journal of Mathematical Analysis and Applications, vol. 311, no. 2, pp. 495-505, 2005.

[13] S. Zhang, "Positive solutions to singular boundary value problem for nonlinear fractional differential equation," Computers \& Mathematics with Applications, vol. 59, no. 3, pp. 1300-1309, 2010.

[14] X.-K. Zhao and W. Ge, "Unbounded solutions for a fractional boundary value problems on the infinite interval," Acta Applicandae Mathematicae, vol. 109, no. 2, pp. 495-505, 2010.

[15] Y. Zhang and Z. Bai, "Existence of solutions for nonlinear fractional three-point boundary value problems at resonance," Journal of Applied Mathematics and Computing, vol. 36, no. 1-2, pp. 417-440, 2011.

[16] W. Jiang, "The existence of solutions to boundary value problems of fractional differential equations at resonance," Nonlinear Analysis: Theory, Methods \& Applications A, vol. 74, no. 5, pp. 1987-1994, 2011.

[17] S. Zhang, "Existence of a solution for the fractional differential equation with nonlinear boundary conditions," Computers \& Mathematics with Applications, vol. 61, no. 4, pp. 1202-1208, 2011.

[18] S. Liang and J. Zhang, "Positive solutions for boundary value problems of nonlinear fractional differential equation," Nonlinear Analysis: Theory, Methods E Applications A, vol. 71, no. 11, pp. 5545-5550, 2009.

[19] Z. Wei, W. Dong, and J. Che, "Periodic boundary value problems for fractional differential equations involving a Riemann-Liouville fractional derivative," Nonlinear Analysis: Theory, Methods E Applications A, vol. 73, no. 10, pp. 3232-3238, 2010.

[20] H. Jafari and V. Daftardar-Gejji, "Positive solutions of nonlinear fractional boundary value problems using Adomian decomposition method," Applied Mathematics and Computation, vol. 180, no. 2, pp. 700-706, 2006.

[21] G. Cerami, "An existence criterion for the critical points on unbounded manifolds," Istituto Lombardo. Accademia di Scienze e Lettere: Rendiconti A, vol. 112, no. 2, pp. 332-336, 1978 (Italian).

[22] P. H. Rabinowitz, "Periodic solutions of Hamiltonian systems," Communications on Pure and Applied Mathematics, vol. 31, no. 2, pp. 157-184, 1978.

[23] J. Mawhin and M. Willem, Critical Point Theory and Hamiltonian Systems, vol. 74 of Applied Mathematical Sciences, Springer, New York, NY, USA, 1989.

[24] P. H. Rabinowitz, Minimax Methods in Critical Point Theory with Applications to Differential Equations, vol. 65 of CBMS Regional Conference Series in Mathematics, American Mathematical Society, Providence, RI, USA, 1986.

[25] G. Fei, "On periodic solutions of superquadratic Hamiltonian systems," Electronic Journal of Differential Equations, vol. 2002, no. 8, pp. 1-12, 2002.

[26] Y. H. Ding and S. X. Luan, "Multiple solutions for a class of nonlinear Schrödinger equations," Journal of Differential Equations, vol. 207, no. 2, pp. 423-457, 2004. 
[27] M. J. Esteban and E. Séré, "Stationary states of the nonlinear Dirac equation: a variational approach," Communications in Mathematical Physics, vol. 171, no. 2, pp. 323-350, 1995.

[28] C.-L. Tang and X.-P. Wu, "Subharmonic solutions for nonautonomous sublinear second order Hamiltonian systems," Journal of Mathematical Analysis and Applications, vol. 304, no. 1, pp. 383-393, 2005.

[29] C.-L. Tang and X.-P. Wu, "Periodic solutions for second order systems with not uniformly coercive potential," Journal of Mathematical Analysis and Applications, vol. 259, no. 2, pp. 386-397, 2001.

[30] C. Troestler and M. Willem, "Nontrivial solution of a semilinear Schrödinger equation," Communications in Partial Differential Equations, vol. 21, no. 9-10, pp. 1431-1449, 1996.

[31] X. Fan and X. Han, "Existence and multiplicity of solutions for $p(x)$-Laplacian equations in $\mathbb{R}^{N}$," Nonlinear Analysis: Theory, Methods E Applications A, vol. 59, no. 1-2, pp. 173-188, 2004.

[32] F. Jiao and Y. Zhou, "Existence of solutions for a class of fractional boundary value problems via critical point theory," Computers \& Mathematics with Applications, vol. 62, no. 3, pp. 1181-1199, 2011.

[33] S. Ma and Y. Zhang, "Existence of infinitely many periodic solutions for ordinary $p$-Laplacian systems," Journal of Mathematical Analysis and Applications, vol. 351, no. 1, pp. 469-479, 2009.

[34] Q. Zhang and C. Liu, "Infinitely many periodic solutions for second order Hamiltonian systems," Journal of Differential Equations, vol. 251, no. 4-5, pp. 816-833, 2011. 


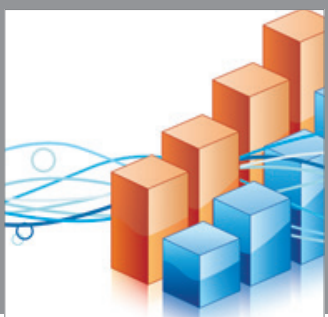

Advances in

Operations Research

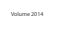

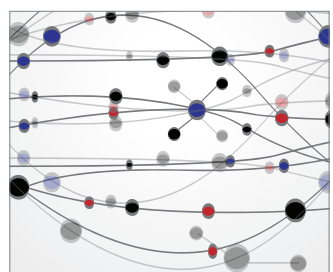

\section{The Scientific} World Journal
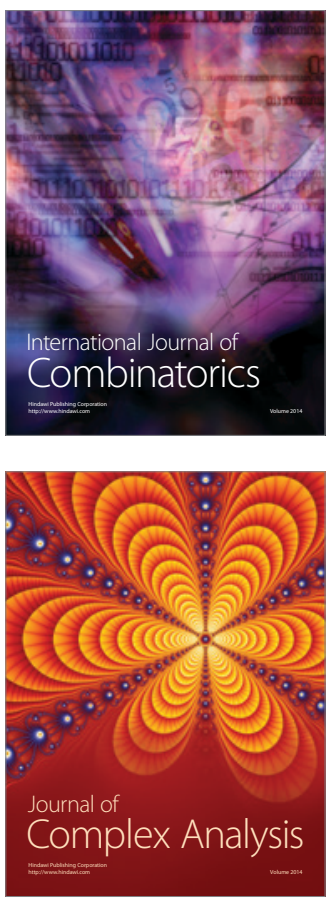

International Journal of

Mathematics and

Mathematical

Sciences
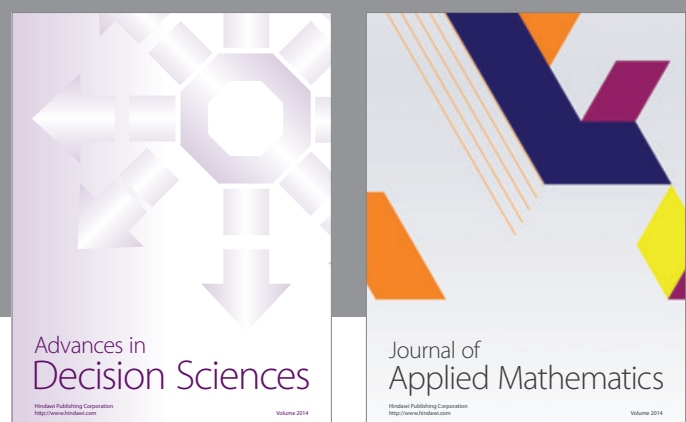

Journal of

Applied Mathematics
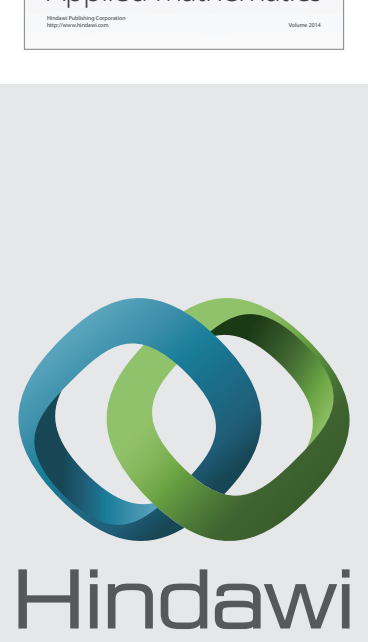

Submit your manuscripts at http://www.hindawi.com
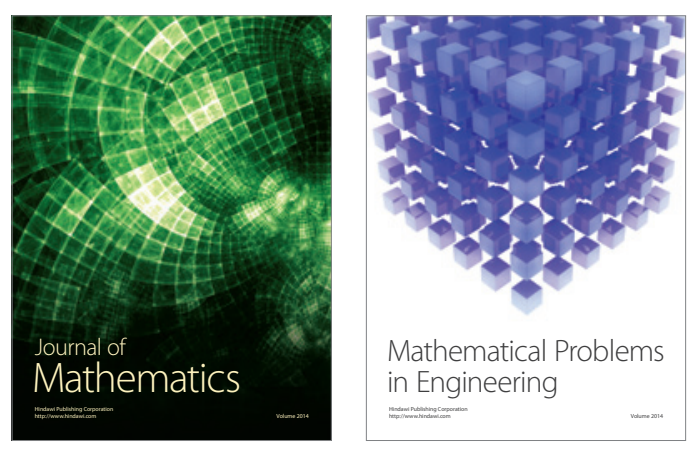

Mathematical Problems in Engineering
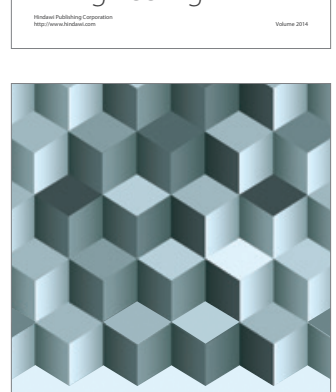

Journal of

Function Spaces
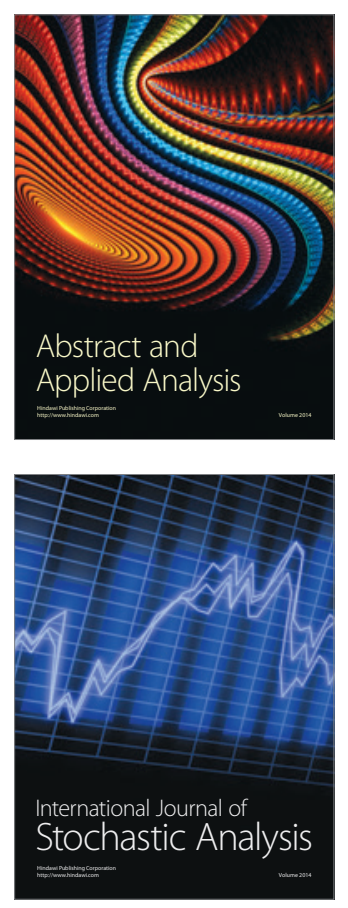

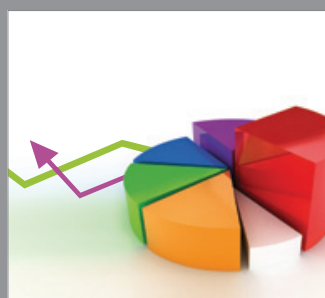

ournal of

Probability and Statistics

Promensencen
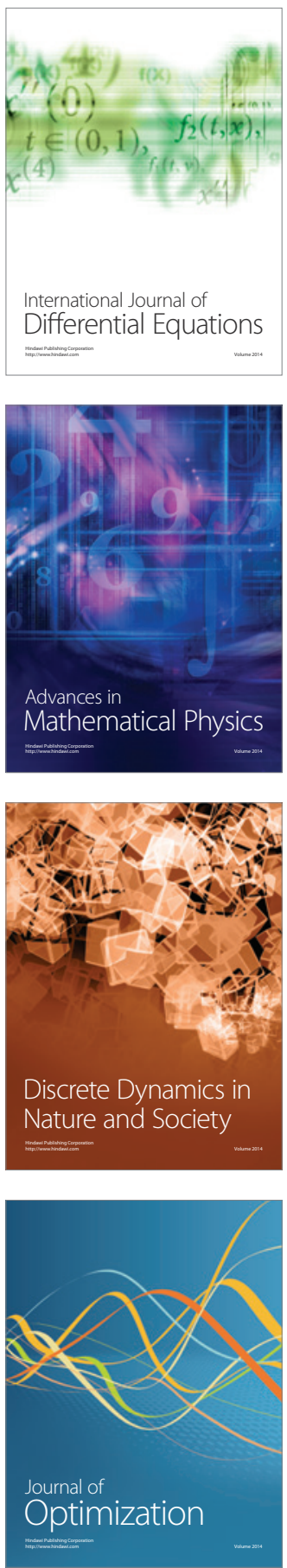